\title{
Low Dose Electron Diffraction Tomography (LD-EDT) in TEM: Application on an hydrated Al arsenate mineral
}

\author{
Emre Yoruk, Stéphanie Kodjikian, Holger Klein \\ CNRS, Institut Néel and Université Grenoble-Alpes - Grenoble - France \\ emre.yoruk@neel.cnrs.fr
}

3D electron diffraction (3D ED) has recently emerged as an alternative to x-ray diffraction to elucidate the atomic structure of nanosized beam sensitive crystals ${ }^{1}$. LD-EDT ${ }^{2}$ is a recently developed low dose 3D ED technique for ab initio structure determination of beam sensitive crystals such as hydrated minerals or MOFs. Low dose conditions are achieved by optimizing exposure during specimen tilting. High quality diffraction data can be obtained from very small crystals without damaging the structure, and a precise sampling of the reciprocal space is assured by beam precession. We recently applied LD-EDT on Bulachite ${ }^{3}$, a hydrated Al arsenate mineral, to solve its atomic structure. Difficulties related to the small size of crystals as well as beam sensitivity due to the presence of $\mathrm{H}_{2} \mathrm{O}$ molecules inside the lattice were overcome by LD-EDT, where synchrotron x-rays previously failed. The resulting structure ${ }^{4}$ is comprised of layers containing edge-sharing Al-O octahedra, inter-connected with As-O tetrahedra by corner sharing. The localization of light atoms in the lattice showcases the potential of electron crystallography for yielding high quality diffraction data even under low dose conditions.

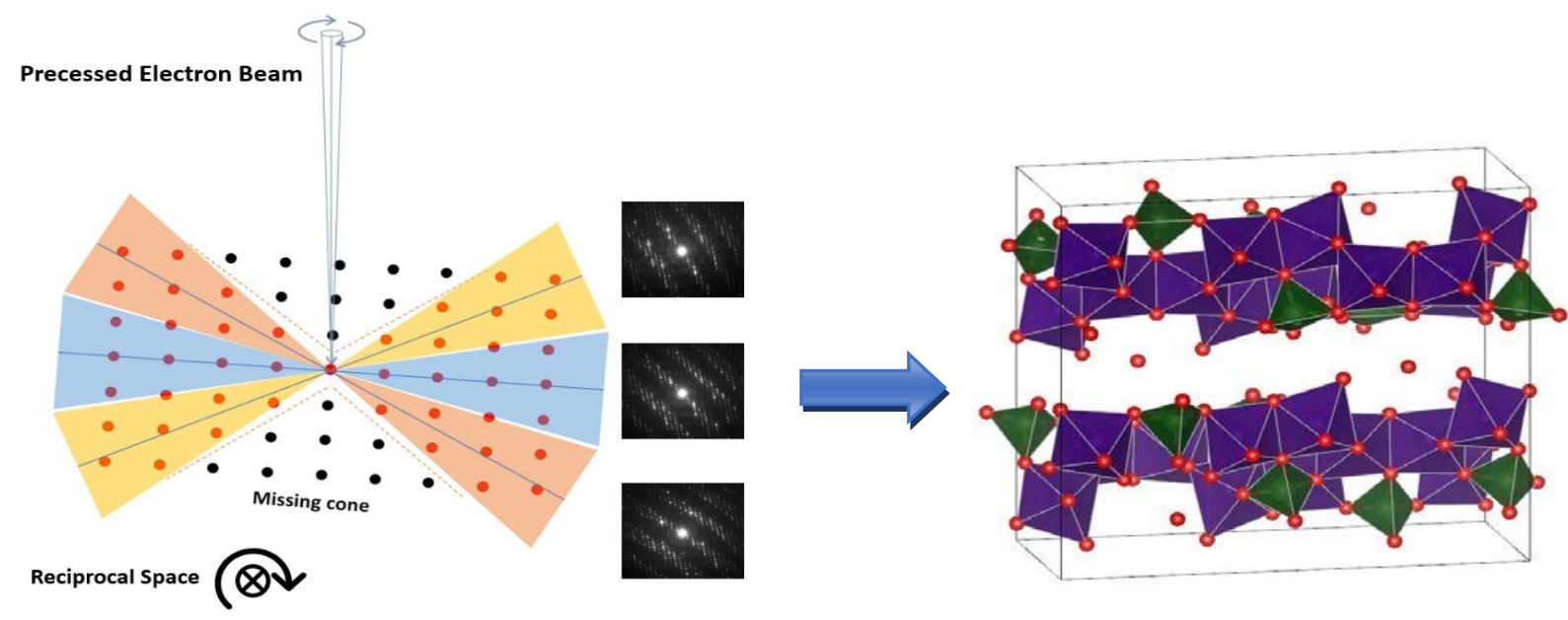

Figure 1: Schematic Representation of data collection in LD-EDT and the obtained atomic structure of Bulachite

1. M. Gemmi et al., ACS Cent. Sci., 2019, 5, 1315-1329.

2 S. Kodjikian, H. Klein, Ultramicroscopy 2019, 200, 12-19.

3. K. Walenta, Aufschluss., 1983, 34, 445-451.

4. I.E. Grey., E. Yoruk et al., Mineralogical Magazine, 2020, 84, 608-615. 